\title{
Fatores associados à mortalidade neonatal em recém-nascidos de baixo peso ao nascer
}

\author{
Factors associated with neonatal mortality in newborns with low birth weight
}

Factores asociados con la mortalidad neonatal en recién nacidos con bajo peso al nacer

Maria Aparecida Munhoz Gaíva ${ }^{1 *}$, Fernanda Sanches Peres Lopes², Leandro Felipe Mufato³, Silvana Margarida Benevides Ferreira ${ }^{1}$.

\section{RESUMO}

Objetivo: Analisar os fatores associados à mortalidade neonatal de recém-nascidos com baixo peso ao nascer. Métodos: Estudo do tipo coorte retrospectiva, com recém-nascidos de baixo peso que foram a óbito nos primeiros 27 dias de vida, no período de 2011 a 2014. Coletou-se dados no Sistema de Informação sobre Nascidos Vivos e Sistema de Informação sobre Mortalidade. O Linkage determinístico unificou os dados dos dois sistemas. Para a análise no efeito de covariáveis sobre a força da mortalidade neonatal em recém-nascidos de baixo peso aplicou-se a regressão simples. E, posteriormente a regressão logística. As variáveis testadas nos modelos de regressão foram selecionadas quando apresentaram a significância estatística para os valores de $p \leq 0,05$, bem como a presença de variáveis de ajuste. Resultados: Mostraram-se como fatores associados à mortalidade neonatal: parto vaginal; realização de 1 a 6 consultas de pré-natal; peso ao nascer entre 500 a 999 gramas; Apgar menor que sete e a presença de anomalia congênita. Conclusão: A ocorrência de óbito neonatal de recém-nascidos com baixo peso se relaciona à qualidade da assistência durante a gestação, ao parto e ao recém-nascido.

Palavras-chave: Mortalidade neonatal, Recém-nascido de baixo peso, Estudos de coortes.

\section{ABSTRACT}

Objective: To analyze the factors associated with neonatal mortality in newborns with low birth weight. Methods: Retrospective cohort study, with low birth weight newborns who died in the first 27 days of life in the period from 2011 to 2014. Data were collected in the Live Birth Information System and Mortality Information System. Deterministic linkage unified the data of the two systems. For the analysis of the effect of covariables on the strength of neonatal mortality in low birth weight newborns, simple regression was applied. And, later, logistic regression. The variables tested in the regression models were selected when they presented statistical significance for $p$ values $\leq 0.05$, as well as the presence of adjustment variables. Results: The following factors were associated with neonatal mortality: male gender; vaginal delivery; 1 to 6 prenatal consultations; birth weight between 500 to 999 grams; Apgar less than seven and the presence of congenital anomaly. Conclusions: The occurrence of neonatal death in newborns with low weight is related to the quality of care during pregnancy, childbirth and the newborn.

Keywords: Infant mortality, Infant low birth weight, Cohort studies.

\section{RESUMEN}

Objetivo: Analizar los factores asociados con la mortalidad neonatal en recién nacidos con bajo peso al nacer. Métodos: Estudio de cohorte retrospectivo, con recién nacidos de bajo peso al nacer que murieron en los primeros 27 días de vida en el período de 2011 a 2014. Los datos se recopilaron en el Sistema de información de nacimientos vivos y el Sistema de información de mortalidad. La vinculación determinista unificó los datos de los dos sistemas. Para el análisis del efecto de las covariables sobre la fuerza de la mortalidad neonatal en recién nacidos de bajo peso al nacer, se aplicó regresión simple. Y, posteriormente, regresión logística. Las variables probadas en los modelos de regresión se seleccionaron cuando presentaban significancia estadística para valores de $p \leq 0.05$, así como la presencia de variables de ajuste. Resultados: Los siguientes factores se asociaron con la mortalidad neonatal: género masculino; parto vaginal; 1 a 6

\footnotetext{
${ }^{1}$ Universidade Federal de Mato Grosso (UFMT), Cuiabá - MT.

${ }^{*}$ E-mail: mamgaiva@gmail.com

2 Universidade Federal de Mato Grosso (UFMT), Barra do Garça - MT

${ }^{3}$ Universidade do Estado de Mato Grosso (UNEMAT), Tangará da Serra - MT.
}

SUBMETIDO EM: 8/2020 ～ACEITO EM: 9/2020 ～～PUBLICADO EM: 11/2020 
consultas prenatales; peso al nacer entre 500 y 999 gramos; Apgar menos de siete y la presencia de anomalía congénita. Conclusión: La aparición de muerte neonatal en recién nacidos con bajo peso está relacionada con la calidad de la atención durante el embarazo, el parto y el recién nacido.

Palabras clave: Mortalidad infantil, Recién nacido de bajo peso, Estudios de cohortes.

\section{INTRODUÇÃO}

O baixo peso ao nascer (BPN) é definido como o peso menor que 2.500 gramas. Dados epidemiológicos mostram que crianças com peso ao nascer inferior a $2500 \mathrm{~g}$ apresentam maior risco de morrer, comparativamente àquelas que nascem com peso adequado (FRANÇA EB, et al., 2017, THE GLOBAL BURDEN OF DISEASE, 2016; LIU L, et al., 2016).

A Organização Mundial da Saúde (OMS) estima que, globalmente, $15 \%$ a $20 \%$ de todos os nascimentos são de baixo peso ao nascer (WHO, 2014). No Brasil, 8,4\% do total de nascidos vivos apresentam baixo peso (LEAL MC, et al., 2018). A sobrevivência desse grupo de recém-nascidos reflete a qualidade da atenção durante a gestação, trabalho de parto e parto, além do atendimento neonatal (BRASIL, 2016).

Todos os anos, nascem aproximadamente 20 milhões de crianças com BPN, em todo o mundo. Deste total, $28 \%$ nascem no Sul da Ásia, $13 \%$ na África subsaariana e $9 \%$ na América Latina (WHO, 2014). As menores taxas de BPN estão presentes em países como Islândia, Finlândia, Noruega e Suécia que apresentam menos de $5 \%$ do total de nascidos vivos (WHO, 2015). Nos países em desenvolvimento, como 0 Brasil, a incidência de BPN varia entre 8,5\% a 12\%, de acordo com a região (WHO, 2014).

Atualmente, no Brasil, os recém-nascidos com BPN representam mais da metade dos óbitos neonatais, especialmente aqueles que nascem com extremo baixo peso (LEAL MC, et al., 2018; BRASIL, 2016; FRANÇA EB, et al., 2016). Apesar da importância do BPN como preditor da mortalidade neonatal, a classificação de BPN não separa os recém-nascidos de baixo peso que são prematuros daqueles que são pequenos para idade gestacional ou daqueles que são prematuros e pequenos para a idade gestacional, fato este que dificulta a identificação dos fatores determinantes da mortalidade nas primeiras quatro semanas de vida (WHO, 2014).

O BPN figura entre as principais características dos recém-nascidos que vieram a óbito no período neonatal, além da prevalência de crianças do sexo masculino. Também é característico da mortalidade neonatal a idade gestacional menor que 37 semanas, baixa vitalidade ao nascer, mães com idade entre 2034 anos que realizaram menos de sete consultas de pré-natal e tiveram parto cesárea; muitas dessas mortes consideradas evitáveis. Assim, a qualidade da atenção obstétrica e neonatal contribui para diminuir características que estão presentes nos casos de óbitos (GAIVA MAM, et al., 2018).

Considerando que o coeficiente de mortalidade neonatal expressa a complexa relação entre fatores biológicos, sociais e da assistência à saúde, o monitoramento dos óbitos neonatais e de seus fatores de risco é fundamental para identificar mudanças e progressos na qualidade dos serviços de saúde materno-infantil, além de serem uteis para direcionar estratégias/ações para redução desse indicador (BORGES TS e VAYEGO AS, 2015, TEIXEIRA GA, et al., 2016, AKINYEMI JO ,et al., 2015).

A despeito do investimento em pesquisas brasileiras enfocando os determinantes da mortalidade neonatal, atualmente poucas abordam estritamente a população de baixo peso ao nascer (OLIVEIRA MG, et al., 2015, CASTRO ECM, et al., 2016). Assim, este estudo tem como objetivo analisar os fatores associados à mortalidade neonatal em recém-nascidos de baixo peso ao nascer em uma capital da região centro-oeste no período de 2011 a 2014

\section{MÉTODOS}

Realizou-se um estudo de coorte retrospectivo, composto por todos os neonatos nascidos vivos em uma capital da região centro-oeste no período de $1^{\circ}$ de janeiro de 2011 a 31 de dezembro de 2014 com peso ao nascer inferior a 2.500 gramas (entre 500 e 2.499 gramas) e que evoluíram a óbito nos primeiros 27 dias de vida, no período de estudo e cujas mães eram residentes no município. 
Foram excluídos do estudo os nascidos vivos com o peso inferior a 500 gramas e portadores de anencefalia. A definição do quadriênio 2011-2014 se deu por ser este período o subsequente a última atualização da Declaração de Nascidos Vivos (DNV). Durante o período de 2011 a 2014, nasceram no município estudado, 39.458 crianças, destas 3.477 foram de baixo peso ao nascer (8,8\%). Do total dos recémnascidos de baixo peso ao nascer, $373(10,7 \%)$ foram a óbito no período neonatal.

Os dados foram obtidos do Sistema de Informação sobre Nascidos Vivos (SINASC) e do Sistema de Informação sobre Mortalidade (SIM), que tem como instrumentos de coleta a Declaração de óbito (DO) e a Declaração de Nascido Vivo (DNV), respectivamente. Os dados foram coletados na Secretaria de Estado de Saúde de Mato Grosso, no Departamento de Vigilância Epidemiológica.

Após a padronização das duas bases de dados foi realizado um linkage determinístico, compondo um único banco de dados. Para o linkage, foi utilizado as variáveis número da DNV; data de nascimento; sexo e idade da mãe. Na presença de divergência entre os dados da DO e a DNV, considerou-se as informações da DNV, já que essas são consideradas de boa qualidade.

Do total de nascidos vivos de baixo peso $(n=3477)$ foram excluídos 73 , sendo 69 com o peso ao nascer inferior a 500 gramas e 4 anencéfalos, perfazendo um total de 3404 neonatos analisados. Desses participantes evoluíram para o óbito 373 crianças, dos quais foram excluídos 135 (36,2\%), sendo 103 com o peso ao nascer igual superior a 2.500 gramas; 4 anencéfalos (3,0\%); 19 com o peso inferior a 500 gramas (14, $1 \%)$; e $9(6,6 \%)$ que não foram concatenados por meio da declaração de nascidos vivos, data de nascimento, sexo e idade materna. Totalizando para análise 238 óbitos e 3166 sobreviventes (Figura 1).

Figura 1 - Fluxograma amostragem da população de RN de BPN vieram á óbito no período neonatal entre 2011 e 2014.

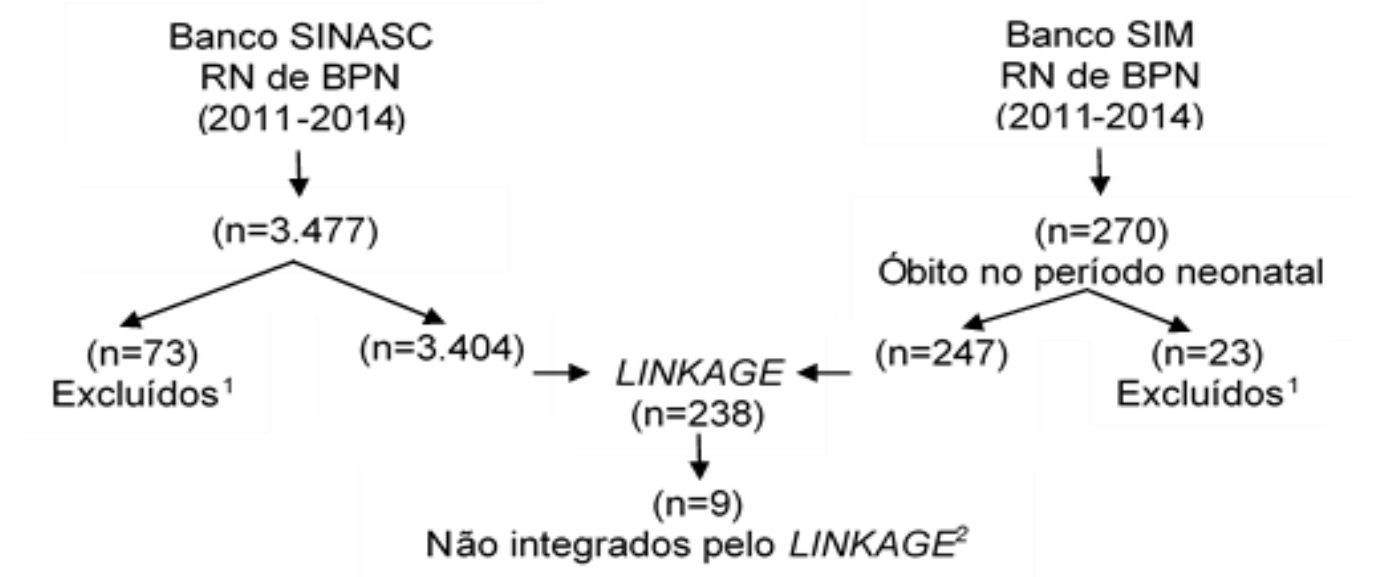

Legenda: ${ }^{1}$ Excluidos do SINASC e SIM os RN de BPN<500g e/ou anencéfalos. No SINASC foram 69 BPN $<500 \mathrm{~g}$ e 4 anencéfalos. ${ }^{2} E r r o$ da data de nascimento, sexo e idade materna.

Fonte: Gaiva MAM, et al., 2020. Baseado em informações do Departamento de Informática da Vigilância Epidemiológica de uma capital da região centro-oeste, Brasil.

Para analisar a mortalidade neonatal de recém-nascidos de baixo peso ao nascer considerou-se como variável dependente aquela dicotomizada em ocorrência de óbito e aqueles que sobreviveram no período neonatal e como variáveis de exposição aquelas relacionadas às condições sociais, biológicas maternas e as variáveis ligadas a presença de malformação congênita ou anomalia cromossômica.

Para a análise do efeito de covariáveis sobre a força da mortalidade neonatal em recém-nascidos de baixo peso aplicou-se a regressão simples. E, posteriormente a regressão logística. As variáveis testadas nos modelos de regressão foram selecionadas quando apresentaram a significância estatística para os valores de $p \leq 0,05$, bem como a presença de variáveis de ajuste. O modelo utilizado na análise multivariada foi o processo Stepwise forward. O programa utilizado foi o software Statistical Package for the Social Sciences for Windows Student Version (SPSS) 17.0. O projeto foi submetido à apreciação do Comitê de Ética e Pesquisa, tendo sido aprovado sob o protocolo $n \div$. 1283.468/2015/CEP-HUJM. 


\section{RESULTADOS}

No total da coorte de nascidos vivos estudado foram identificados 373 óbitos neonatais de recém-nascidos de BPN em uma capital da região centro-oeste. A probabilidade de morrer antes de completar 28 dias de vida foi de $9,4 \%$ para cada mil nascidos vivos. Do total de 238 óbitos neonatais analisados no presente estudo, $177(74,4 \%)$ ocorreram no período neonatal precoce (nos primeiros seis dias de vida) e 61 (25,6\%), no período neonatal tardio (do sétimo a 27 dias de vida).

Observou-se, na análise bivariada que: o risco do recém-nascido BPN evoluir ao óbito é 30,6\% maior nos nascimentos de parto normal em relação aos por cesariana $(R R=1,31 \mathrm{IC}=95 \% 1,02 ; 1,67)$; aqueles que não tiveram nenhuma consulta de pré-natal ( $R R=4,09$; IC 95\% 2,28-7,35) e nascidos vivos de mães que realizaram de uma a seis consultas ( $R R=2,56$; IC 95\% 1,95-3,37) tiveram maior risco de evoluir ao óbito em relação aqueles cujas mães tiveram sete ou mais consultas de acompanhamento; aquelas crianças que nasceram com menos de 37 semanas que foram a óbito apresentaram o risco de quase 8 vezes $(R R=7,89$ $\mathrm{IC}=95 \% 4,75 ; 13,01)$ maior do que os recém-nascidos de BPN com maior de 37 semanas de período gestacional.

Os nascidos vivos de BPN de mães com idade inferior a vinte anos ( $R R=1,61 \mathrm{IC}_{95 \%}$ 1,03;2,53) tiveram maior risco de óbito quando comparados aos nascidos vivos de BPN de mães com a idade igual ou superior 35 anos (Tabela 1).

Tabela 1 - Análise bivariada da distribuição de óbitos neonatais e dos recém-nascidos sobreviventes com baixo peso ao nascer segundo variáveis assistenciais.

\begin{tabular}{|c|c|c|c|c|c|c|c|}
\hline \multirow{2}{*}{ Variável Assistencial } & \multicolumn{4}{|c|}{$\begin{array}{c}\text { Óbitos } \\
\text { Sobrevivente }\end{array}$} & \multirow[t]{2}{*}{ RR bruto } & \multirow[t]{2}{*}{ IC $95 \%$} & \multirow[t]{2}{*}{ Valor de $p$} \\
\hline & $\mathbf{n}$ & $\%$ & $\mathbf{n}$ & $\%$ & & & \\
\hline \multicolumn{8}{|c|}{ Tipo de parto $(n=3404)$} \\
\hline Vaginal & 101 & 42,5 & 1127 & 35,6 & 1,31 & 1,$02 ; 1,67$ & 0,034 \\
\hline Cesário & 137 & 57,5 & 2039 & 64,4 & 1 & - & - \\
\hline \multicolumn{8}{|c|}{ Tipo de gravidez $(n=3399)^{*}$} \\
\hline Única & 196 & 82,4 & 2601 & 82,2 & 1,00 & 0,$73 ; 1,38$ & 0,979 \\
\hline Múltipla & 42 & 17,6 & 560 & 17,7 & 1 & - & - \\
\hline \multicolumn{8}{|c|}{ Consulta Pré-natal $(n=3301)^{*}$} \\
\hline Nenhuma & 11 & 4,9 & 57 & 1,9 & 4,09 & 2,$28 ; 7,35$ & $<0,0001$ \\
\hline 1 a 6 & 138 & 61,9 & 1223 & 39,7 & 2,56 & 1,$95 ; 3,37$ & $<0,0001$ \\
\hline$\geq 7$ & 74 & 33,2 & 1798 & 58,4 & 1 & - & - \\
\hline \multicolumn{8}{|c|}{ Duração da gestação $(n=3338)^{*}$} \\
\hline$<37$ & 214 & 93,0 & 1888 & 60,7 & 7,86 & 4,$75 ; 13,01$ & $<0,0001$ \\
\hline$\geq 37$ & 16 & 7,0 & 1220 & 39,3 & 1 & - & - \\
\hline \multicolumn{8}{|c|}{ Local de nascimento $(n=3404)$} \\
\hline Hospital & 235 & 98,7 & 3141 & 99,2 & 0,65 & 0,$22 ; 1,91$ & 0,437 \\
\hline Outros & 3 & 1,3 & 25 & 0,8 & 1 & - & - \\
\hline \multicolumn{8}{|c|}{ Idade maternal $(\mathrm{n}=3404)$} \\
\hline$<20$ & 56 & 23,5 & 528 & 16,7 & 1,61 & 1,$03 ; 2,53$ & 0,033 \\
\hline $20-34$ & 156 & 65,6 & 2226 & 70,3 & 1,10 & 0,$74 ; 1,65$ & 0,631 \\
\hline$\geq 35$ & 26 & 10,9 & 412 & 13,0 & 1 & - & - \\
\hline \multicolumn{8}{|l|}{ Cor/raça $(n=3374)$} \\
\hline Preta & 12 & 5,1 & 184 & 5,9 & 0,81 & 0,$47 ; 1,46$ & 0,514 \\
\hline Amarela & 1 & 0,4 & 19 & 0,6 & 0,68 & 0,$10 ; 4,60$ & 0,685 \\
\hline Branca & 51 & 21,4 & 750 & 23,9 & 0,86 & 0,$64 ; 1,17$ & 0,335 \\
\hline Parda & 174 & 73,1 & 2183 & 69,6 & 1 & - & - \\
\hline Total ** & 238 & 7,0 & 3166 & 3404 & 100 & - & - \\
\hline
\end{tabular}

Legenda: *Excluídos os ignorados; ${ }^{* *}$ Cálculo em linha do total dos participantes estudados.

Fonte: Gaiva MAM, et al., 2020. Baseado em informações do Departamento de Informática da Vigilância Epidemiológica de uma capital da região centro-oeste, Brasil. 
Crianças do sexo masculino apresentaram $46,4 \%$ de chance de irem a óbito no período neonatal quando comparadas as do sexo feminino ( $R R=1,46$ IC $95 \%$ 1,14-1,87). Nascidos vivos de BPN com o peso entre 500 a 999 gramas $\left(R R=22,36\right.$; $\left.I_{95 \%} 17,18 ; 29,10\right)$ e nascidos vivos de $B P N$ com o peso igual a 1.000 a 1499 gramas (RR=6,61; IC 95\% $_{4,70 ; 9,30)}$ tiveram maior probabilidade de evoluírem ao óbito em relação aos que tiveram peso entre 1.500 a 2.499 gramas.

Neonatos de BPN que apresentaram Apgar inferior a sete no primeiro (RR 14,33; IC $95 \%$ 10,14-20,27) e quinto minutos de vida (RR 10,64; $\mathrm{IC}_{95 \%} 8,43 ; 13,43$ ) e aqueles com a presença de malformação congênita (RR 5,70; IC $95 \% 4,14 ; 7,85$ ) mostraram-se associadas à mortalidade neonatal de recém-nascidos de BPN (Tabela 2).

Tabela 2 - Análise bivariada da distribuição de óbitos neonatais e dos recém-nascidos sobreviventes com baixo peso ao nascer segundo as características biológicas do recém-nascido.

\begin{tabular}{|c|c|c|c|c|c|c|c|}
\hline \multirow{3}{*}{$\begin{array}{c}\text { Variável biológica do recém- } \\
\text { nascido }\end{array}$} & \multicolumn{4}{|c|}{$\begin{array}{l}\text { Recém-nascido com baixo } \\
\text { peso ao nascer }\end{array}$} & \multirow{3}{*}{$\begin{array}{c}\text { RR } \\
\text { bruto }\end{array}$} & \multirow{3}{*}{ IC $95 \%$} & \multirow{3}{*}{$\begin{array}{l}\text { valor } \\
\text { de } p\end{array}$} \\
\hline & \multicolumn{2}{|c|}{ Óbitos } & \multicolumn{2}{|c|}{$\begin{array}{c}\text { Sobrevivent } \\
\text { e }\end{array}$} & & & \\
\hline & $\mathbf{n}$ & $\%$ & $\mathbf{n}$ & $\%$ & & & \\
\hline \multicolumn{8}{|l|}{$\operatorname{Sexo}(n=3403)^{*}$} \\
\hline Masculino & 131 & 55,3 & 1427 & 45,1 & 1,46 & $(1,14 ; 1,87)$ & 0,002 \\
\hline Feminino & 106 & 44,7 & 1739 & 54,9 & 1 & - & - \\
\hline \multicolumn{8}{|l|}{ Peso ao nascer (gramas) $(n=3404)$} \\
\hline 500 a 999 & 117 & 49,2 & 100 & 3,1 & 22,36 & $(17,18 ; 29,10)$ & $<0,001$ \\
\hline 1.000 a 1.499 & 52 & 21,8 & 274 & 8,7 & 6,61 & $(4,70 ; 9,30)$ & $<0,001$ \\
\hline 1.500 a 2.499 & 69 & 29,0 & 2792 & 88,2 & 1 & - & - \\
\hline \multicolumn{8}{|l|}{ Apgar 1을 minuto $(n=3391)^{*}$} \\
\hline$\leq 7$ & 202 & 84,9 & 752 & 23,9 & 14,33 & $(10,14 ; 20,27)$ & $<0,001$ \\
\hline$\geq 8$ a 10 & 36 & 15,1 & 2401 & 76,1 & 1 & - & - \\
\hline \multicolumn{8}{|l|}{ Apgar 5o minuto $(n=3392)^{\star}$} \\
\hline$\leq 7$ & 135 & 56,7 & 237 & 7,5 & 10,64 & $(8,43 ; 13,43)$ & $<0,001$ \\
\hline$\geq 8$ a 10 & 103 & 43,3 & 2917 & 92,5 & 1 & - & - \\
\hline \multicolumn{8}{|l|}{ Presença de anomalia $(n=3392)^{*}$} \\
\hline Sim & 29 & 12,2 & 52 & 1,6 & 5,70 & $(4,14 ; 7,85)$ & $<0,001$ \\
\hline Não & 208 & 87,8 & 3103 & 98,4 & 1 & - & - \\
\hline Total $^{* *}$ & 238 & 7,0 & 3166 & 3404 & 100 & - & - \\
\hline
\end{tabular}

Legenda: *Excluídos os ignorados; ${ }^{* *}$ Cálculo em linha do total dos participantes estudados.

Fonte: Gaiva MAM, et al., 2020. Baseado em informações do Departamento de Informática da Vigilância Epidemiológica de uma capital da região centro-oeste, Brasil.

As variáveis relativas às características sociais (escolaridade materna, situação conjugal materna e ocupação materna), assistenciais (tipo de gravidez e local de nascimento), biológicas materna (cor/raça) não apresentaram diferenças estatisticamente significantes na análise bivariada, com a mortalidade neonatal de recém-nascidos de BPN.

Verificou-se que o risco de óbito entre os recém-nascidos masculinos foi 2 vezes maior $(\mathrm{OR}=2,34$ IC95\% $1,04 ; 1,63)$ do que os neonatos femininos; nascidos vivos de BPN por parto vaginal tiveram o risco de óbito 2 vezes maior quando comparados aos nascidos por parto cesário (OR=2,2 IC $\left.{ }_{95} \% 1,03 ; 1,52\right)$; nascidos vivos de mães que realizaram uma a seis consultas de pré-natal $\left(\mathrm{OR}=2,52 ; \mathrm{IC}_{95 \%}\right.$ 1,08-1,83) tiveram maior risco de ir ao óbito em relação as mães que realizaram mais de sete consultas; o risco de óbito neonatal é 9 vezes maior naqueles recém-nascidos com o peso entre 500 a 999 gramas e 5 vezes maior naqueles recémnascidos com o peso entre 1.000 a 1.499 gramas quando comparados ao peso entre 1.500 a 2.499 gramas; bebês com Apgar igual ou inferior a sete no primeiro e no quinto minuto de vida apresentaram mais chances 
de evoluírem a óbito ( $\mathrm{OR}=6,28$; $\left.\mathrm{IC}_{95 \%} 2,61-6,21\right)$, (OR=3,34; IC $95 \%$ 1,22-2,13) respectivamente; neonatos com malformação congênita apresentaram 6 vezes mais chances de evoluírem ao óbito quando comparados aos que não apresentavam anomalia $\left(\mathrm{OR}=5,9 ; \mathrm{IC}_{95 \%} 2,11-4,39\right)$. $\mathrm{O}$ modelo final dos fatores associados à mortalidade neonatal de baixo peso ao nascer está apresentado na Tabela 3.

Tabela 3 - Fatores de risco à mortalidade neonatal de recém-nascidos de baixo peso ao nascer após ajustamento.

\begin{tabular}{|c|c|c|c|}
\hline \multirow{2}{*}{ Variáveis } & \multicolumn{2}{|c|}{ Análise múltipla } & \multirow{2}{*}{ Valor $p$} \\
\hline & OR ajustado ${ }^{a}$ & IC 95\% & \\
\hline \multicolumn{4}{|l|}{ Sexo } \\
\hline Masculino & 2,34 & $(1,04 ; 1,63)$ & 0,020 \\
\hline Feminino & 1 & - & - \\
\hline \multicolumn{4}{|c|}{ Duração da gestação (semanas) } \\
\hline$\leq 37$ & 1,66 & $(0,93 ; 2,40)$ & 0,098 \\
\hline$>37$ & 1 & - & - \\
\hline \multicolumn{4}{|l|}{ Tipo de parto } \\
\hline Vaginal & 2,21 & $(1,03 ; 1,52)$ & 0,019 \\
\hline Cesáreo & 1 & - & - \\
\hline \multicolumn{4}{|c|}{ № consultas de pré-natal } \\
\hline Nenhuma & 0,58 & $(0,72 ; 1,83)$ & 0,564 \\
\hline 1 a 6 consultas & 2,52 & $(1,08 ; 1,83)$ & 0,012 \\
\hline$\geq 7$ consultas & 1 & - & - \\
\hline \multicolumn{4}{|c|}{ Peso ao nascer (gramas) } \\
\hline $500 \mathrm{a} \leq 999$ & 9,74 & $(4,72 ; 10,30)$ & 0,000 \\
\hline $1.000 \mathrm{a} \leq 1.499$ & 5,15 & $(1,94 ; 4,37)$ & 0,000 \\
\hline $1.500 \mathrm{a} \leq 2.499$ & 1 & - & - \\
\hline \multicolumn{4}{|c|}{ Idade materna (anos) } \\
\hline$<20$ & 1,46 & $(0,89 ; 2,16)$ & 0,143 \\
\hline $20-34$ & 1,42 & $(0,89 ; 1,99)$ & 0,156 \\
\hline$\geq 35$ & 1 & - & - \\
\hline \multicolumn{4}{|l|}{ Apgar 1ํ minuto } \\
\hline$\leq 7$ & 6,28 & $(2,61 ; 6,21)$ & 0,000 \\
\hline$\geq 8$ a 10 & 1 & - & - \\
\hline \multicolumn{4}{|l|}{ Apgar 5 minuto } \\
\hline$\leq 7$ & 3,34 & $(1,22 ; 2,13)$ & 0,001 \\
\hline$\geq 8$ a 10 & 1 & - & - \\
\hline \multicolumn{4}{|c|}{ Presença de anomalia } \\
\hline Sim & 5,95 & $(2,11 ; 4,39)$ & 0,000 \\
\hline Não & 1 & - & - \\
\hline
\end{tabular}

Legenda: ${ }^{a}$ Odds ratio ajustada para todas as variáveis através das variáveis cor/raça, escolaridade da mãe, situação conjugal e tipo de gravidez.

Fonte: Gaiva MAM, et al., 2020. Baseado em informações do Departamento de Informática da Vigilância Epidemiológica de uma capital da região centro-oeste, Brasil. 


\section{DISCUSSÃO}

Nesta coorte foi observado o predomínio o óbito neonatal na primeira semana de vida $(74,4 \%)$, sendo que $41,2 \%$ destes ocorreram nas primeiras 24 horas, semelhante a outros estudos (CASTRO ECM, et al., 2016; TEIXEIRA GA et al., 2016; BAQUI AH, et al., 2016; MOGHADDAM PS, AGHAALI M, 2015). Sabe-se que os óbitos neonatais precoces estão relacionados às condições da assistência no período do pré-parto e ao parto (CASTRO ECM, et al., 2016).

Desta maneira, a alta proporção de óbitos precoces deve ser vista com atenção por gestores e profissionais de saúde em razão de que as questões relacionadas com a atenção materno-infantil interferem diretamente na sobrevivência da criança, a despeito da influência dos fatores biológicos e sociais.

No presente estudo, mostraram-se como associados ao óbito neonatal as variáveis ligadas a qualidade da assistência (número de consultas de pré-natal, duração da gestação), aquelas relacionadas às variáveis biológicas dos neonatos (sexo masculino, peso ao nascer e a presença de malformações congênitas) e as que refletem a qualidade da assistência ao trabalho de parto e parto (Apgar no $1^{\circ}$ e $5^{\circ}$ minuto de vida menor que 7).

Os recém-nascidos do sexo masculino tiveram maior ocorrência de morte em comparação aos do sexo feminino, o que corrobora com outros estudos (CASTRO ECM, et al., 2016; DIAS BAS, et al., 2019). Tal achado pode estar relacionado às condições associadas ao amadurecimento tardio do pulmão verificado em maior prevalência no sexo masculino. Quanto a idade gestacional das mães dos neonatos, observou-se maior prevalência $(90 \%)$ de ocorrência de risco de morte entre os prematuros com idade gestacional menor de 37 semanas.

Outros estudos indicam, como na presente pesquisa, altas taxas mortalidade entre crianças prematuras (LEAL MC, et al., 2018; TEIXEIRA GA, et al., 2016; CASTRO ECM, et al., 2016; TEIXEIRA JAM, et al., 2019; SLEUTIES FCM, et al., 2018; FILHO ACAA, et al., 2017). Acrescido a prematuridade, encontra-se a condição dos recém-nascidos com muito baixo peso ao nascer (SETUMBA MJ, et al., 2018).

A etiologia do BPN é multifatorial e pode estar relacionada à condição socioeconômica, a educação materna, a renda familiar e desigualdade no acesso ao pré-natal (LEAL MC et al., 2018), além de fatores obstétricos, morbidades maternas e cuidados durante a gravidez (SETUMBA MJ, et al., 2018). Alguns desses fatores podem ser modificáveis com uma assistência à saúde materna de qualidade, precoce e contínua.

O peso ao nascer é considerado um indicador de prognóstico de morte neonatal, sendo que o BPN é o contribuinte mais relevante para o óbito (LEAL MC et al., 2018; TEIXEIRA GA, et al., 2019; SLEUTIES FCM, et al., 2018). As pesquisas mostram que as maiores taxas de mortes neonatais ocorrem entre os neonatos com peso ao nascer menor que 1000g (CASTRO ECM, et al., 2016; SETUMBA MJ, et al., 2018), reafirmando que quanto menor o peso de nascimento maiores as chances de óbito neonatal.

Apesar das evidências cientificas indicarem, alta prevalência dos óbitos neonatais em crianças de baixo peso, ou ainda, nas com extremo baixo peso, há uma tendência de redução desse indicador nas últimas décadas, o que sugere, dentre outros fatores, melhoria da qualidade da assistência ligada a oferta de corticoides e surfactante nas unidades neonatais e melhores condições de vida.

A presença de malformação congênita na presente investigação mostrou-se associada ao desfecho, sendo que os neonatos com esse agravo apresentaram chance de morte neonatal seis vezes maior do que aqueles sem malformação. Nos anos de 1990, as anomalias congênitas eram a quinta causa de mortalidade infantil no Brasil e atualmente representam a segunda causa de óbito (FRANÇA EB et al., 2016). Agravos esses considerados não evitáveis e de difícil redução.

Em países desenvolvidos e com menores taxas de mortalidade infantil as malformações congênitas são as principais causas de morte (TEIXEIRA GA, et al., 2016), sendo que no Japão elas são a primeira causa de óbito (KOSHIDA S, et al., 2016). Ressalta-se que apesar da maioria das malformações ser detectáveis ao nascer, algumas delas necessitam de avaliação e diagnóstico posterior, o que pode levar a subnotificações na DNV. 
Fatores como idade e escolaridade materna e raça marcam padrões de iniquidades expressos através de desfechos evitáveis. No presente estudo as variáveis sociais não se associaram ao óbito neonatal dos RNBPN, apesar de reconhecidamente os determinantes sociais terem influência nos nascimentos prematuros e de baixo peso. Entre estes fatores destacam-se a idade materna, baixa escolaridade das mães e condições desfavoráveis de vida (LEAL MC, et al., 2018; LEAL MD, et al., 2016).

Há que se destacar a limitação da análise de alguns desses determinantes nas pesquisas que utilizam como fonte de dados o SINASC e SIM, uma vez que esses sistemas não contêm variáveis que deem conta dessa análise. Apesar da não associação com a mortalidade neonatal em recém-nascidos de BPN, esses fatores não devem ser desconsiderados, já que constituem uma rede causal na determinação do óbito neonatal, e podem ter sido mascarados pelo número de variáveis analisadas, tamanho da população e ainda pelas características socioeconômicas do município estudado. Das variáveis assistenciais analisadas apresentaram associação com o desfecho o tipo de parto, número de consultas de acompanhamento da gestação e índice de Apgar no $1^{\circ}$ e $5^{\circ}$ minuto de vida. O parto normal apresentou-se como fator associado à mortalidade neonatal, semelhante a outras pesquisas nacionais e internacionais (TEIXEIRA JAM, et al., 2019; MOGHADDAM PS e AGHAALI M, 2015; MUGO NS, et al., 2019).

No presente estudo o parto cesário sugeriu proteção à mortalidade neonatal, assim como outra investigação desenvolvida em capital do nordeste brasileiro (GONZAGA ICA, et al., 2016). Essa proteção pode ter ocorrido por tratar de estudo com uma população de risco para o nascimento precoce, e que está sujeita a complicações clínico obstétricas que podem indicar a necessidade do parto operatório (SANDERS LSC, et al., 2017).

A insuficiência de consultas de acompanhamento pré-natal apresentou forte associação com o óbito de recém-nascido BPN neste estudo, o que já foi evidenciado em outras investigações (CASTRO ECM, et al., 2016; SLEUTIES FCM, et al., 2018; SANDERS LSC, et al., 2017), a exemplo também da pesquisa desenvolvida em uma cidade de Angola na África com dados secundários de 2.100 bebês de baixo peso ao nascer, em que os altos índices de mortalidade neonatal relacionaram-se ao acompanhamento pré-natal inadequado (menos de 6 consultas) (SETUMBA MJ, et al., 2018).

O número mínimo de sete consultas tem efeito protetor sobre a gestação, uma vez que permite a implementação de condutas para a detecção e tratamento de agravos e orientações sobre o processo gravídico-puerperal, possibilitando uma assistência de qualidade durante a gravidez.O Apgar no primeiro e quinto minuto menor que sete constituiu em fator de risco para o óbito neonatal no presente estudo, com chance elevada para sua ocorrência. Resultado esse já descrito em outra pesquisa realizada na região nordeste do país com recém-nascidos de baixo peso (CASTRO ECM, et al., 2016).Investigação que avaliou os determinantes e resultados perinatais de recém-nascidos de baixo peso nascidos no Hospital Especializado Integral da Universidade de Hawassa, no sul da Etiópia, mostrou que os neonatos com BPN tem risco aumentado de apresentar baixo índice de Apgar (TADESE M, et al., 2019), assim como mostrou a pesquisa que analisou a mortalidade neonatal em recém-nascidos angolanos de baixo peso ao nascer (SETUMBA MJ, et al., 2018).

O Apgar do primeiro minuto está relacionado às condições da gestação e do parto, já o do quinto minuto tem relação com a assistência prestada durante o parto e pós-parto, além de sofrer influência de fatores que atuam antes mesmo do nascimento. No entanto, este escore não deve ser analisado de forma isolada, visto que a ocorrência do desfecho negativo é resultante de fatores biológicos, sociais e inclusive aqueles atinentes a assistência a que a criança está submetida.

Sabe-se que a qualidade das informações do SINASC e SIM é essencial para aumento de robustez de uma investigação científica, com menor possibilidade de vieses na análise dos dados. $O$ déficit de preenchimento de algumas variáveis (situação conjugal, $n^{\circ}$ - de consultas de pré-natal e duração da gestação) nas DNV pode ser uma limitação de estudos que utilizam dados secundários. Outro fator limitante da pesquisa foi a não realização da análise da natureza jurídica (privado/público) e a complexidade do hospital de nascimento da criança, já que estudos mostram que nascer em estabelecimento de saúde sem UTI Neonatal constitui um fator de risco para o óbito. Tais indicadores não foram passiveis de análise dado a indisponibilidade dos mesmos nos sistemas de informações utilizados. 


\section{CONCLUSÃO}

Os fatores que se associaram a mortalidade neonatal de crianças de baixo peso ao nascer foram: número de consultas de pré-natal menor que sete, peso ao nascer entre 500 a 999 gramas, Apgar no primeiro e no quinto minuto de vida menor que sete e a presença de anomalia congênita. Os achados, do presente estudo, indicam que os fatores preditores de ocorrência de mortalidade neonatal estão relacionados com a atenção à saúde da gestante e do recém-nascido, em sua maioria evitáveis por adequada atenção dos serviços de saúde. Recomenda-se aos gestores e profissionais da saúde, especial atenção para a qualidade da assistência perinatal oferecida pelo município.

\section{REFERÊNCIAS}

1. AKINYEMI JO, et al. Trends in neonatal mortality in Nigeria and effects of bio-demographic and maternal characteristics. BMC Pediatrics, 2015; 15(36):1-12.

2. BAQUI AH, et al. Neonatal mortality within 24 hours of birth in six low-and lower-middle-income countries. Bulletin World Health Organ, 2016; 94(10):752-8B.

3. BORGES TS, VAYEGO AS. Fatores de risco para a mortalidade neonatal em um município na região Sul. Ciência \& Saúde, 2015; 8(1):7-14.

4. BRASIL. Ministério da Saúde. Secretaria de Ciência, Tecnologia. Secretaria de Ciência, Tecnologia e Insumos Estratégicos. Departamento de Ciência e Tecnologia. Síntese de evidências para políticas de saúde: mortalidade perinatal. 2. ed. Brasília: Ministério da Saúde, 2016.

5. CASTRO ECM, et al. Mortalidade com 24 horas de vida de recém-nascidos pré-termo de muito baixo da Região Nordeste do Brasil. Rev paul pediatr, 2016;34(1):106-113.

6. DIAS BAS, et al. Análise espacial dos óbitos infantis evitáveis no Espírito Santo, Brasil, 2006-2013. Revista Epidemiologia e Serviços de Saúde, 2019; 28(3):e2018111.

7. FILHO ACAA, et al. Aspectos epidemiológicos da mortalidade neonatal em capital do nordeste do Brasil. Revista Cuidarte, 2017; 8(3):1767-76.

8. FRANÇA EB, et al. Principais causas da mortalidade na infância no Brasil, em 1990 e 2015: estimativas do estudo de Carga Global de Doença. Revista Brasileira de Epidemiologia, 2017; 20 (Suppl 2):46-60.

9. GAÍVA MAM, et al. Neonatal deaths of low birth weight newborns. Revista Eletrônica de Enfermagem, 2018; 20:v20a18.

10. GONZAGA ICA, et al. Atenção pré-natal e fatores de risco associados à prematuridade e baixo peso ao nascer em capital do nordeste brasileiro. Ciência \& Saúde Coletiva, 2016; 21(6):1965-1974.

11. KOSHIDA S, et al. Possible Prevention of Neonatal Death: A Regional Population-Based Study in Japan. Yonsei Medical Journal, 2016; 57(2):426-429.

12. LEAL MC, et al. Saúde reprodutiva, materna, neonatal e infantil nos 30 anos do Sistema Único de Saúde (SUS). Ciência \& Saúde Coletiva, 2018; 23(6):1915-28.

13. LEAL MD, et al. Prevalence and risk factors related to preterm birth in Brazil. Reproductive Health. 2016; 13(Suppl 3):127.

14. LIU L, et al. Global, regional, and national causes of under-5 mortality in 2000-15: an updated systematic analysis with implications for the Sustainable Development Goals. Lancet, 2016; 388(10063):3027-35.

15. MOGHADDAM PS, AGHAALI M. Survival of 798 low birth weight infants according to birth weight and gestational age. Shiraz E-Medical Journal. 2015; 16(11,12):e31761.

16. MUGO NS, et al. Exploring causal pathways for factors associated with neonatal, infant and under-five mortality, analysis of 2015-2016 Myanmar Demographic Health Survey. Journal of Global Health Reports, 2019; 3:e2019015.

17. OLIVEIRA MG, et al. Mortalidade neonatal precoce em recém-nascidos de muito baixo peso: estudo de coorte. Boletim Científico de Pediatria, 2015; 4(3):75-9.

18. SANDERS LSC, et al. Mortalidade infantil: análise de fatores associados em uma capital do Nordeste brasileiro. Cadernos Saúde Coletiva, 2017; 25(1):83-9.

19. SETUMBA MJ, et al. Mortalidade em recém-nascidos de baixo peso ao nascer: limites e desafios para o acesso universal. Portuguese Journal of Public Health, 2018; 36:95-101.

20. SLEUTJES FCM, et al. Fatores de risco de óbito neonatal em região do interior paulista, Brasil. Ciência \& Saúde Coletiva, 2018; 23(8):2713-2720.

21. TADESE M, et al. Determinants and adverse perinatal outcomes of low birth weight newborns delivered in Hawassa University Comprehensive Specialized Hospital, Ethiopia: a cohort study. BMC Research Notes, 2019; $12: 118$.

22. TEIXEIRA GA, et al. Fatores de risco para a mortalidade neonatal na primeira semana de vida. Revista de Pesquisa: Cuidado é Fundamental, 2016; 8(1):4036-4046.

23. TEIXEIRA JAM, et al. Mortality in the first day of life: trends, causes of death and avoidability in eight Brazilian Federative Units, between 2010 and 2015. Revista Epidemiolologia e Serviços de Saúde, 2019; 28(1):e2018132.

24. THE GLOBAL BURDEN OF DISEASE 2015 Child Mortality Collaborators. Global, regional, national, and selected subnational levels of stillbirths, neonatal, infant, and under-5 mortality, 1980-2015: a systematic analysis for the Global Burden of Disease Study 2015. Lancet, 2016; 388(10053):1725-74.

25. WORLD HEALTH ORGANIZATION (WHO). World Health Statistics 2015. Genebra; 2015.

26. WORLD HEALTH ORGANIZATION. Global nutrition targets 2025: childhood overweight policy brief. Geneva; 2014. 